\title{
Resolving Active Sites in Atomically Dispersed Electrocatalysts for Energy Conversion Applications
}

\author{
David A. Cullen ${ }^{1, *}$, Karren L. More ${ }^{1}$, K.C. Neyerlin², Hoon T. Chung ${ }^{3}$, Piotr Zelenay ${ }^{3}$, and Deborah J. \\ Myers $^{4}$ \\ 1. Center of Nanophase Materials Sciences, Oak Ridge National Laboratory, Oak Ridge, TN USA. \\ 2. Chemistry and Nanoscience Department, National Renewable Energy Laboratory, Golden, CO USA. \\ 3. Materials Physics \& Applications Division, Los Alamos National Laboratory, Los Alamos, NM USA. \\ 4. Chemical Sciences \& Engineering Division, Argonne National Laboratory, Argonne, IL USA. \\ * Corresponding author: cullenda@ornl.gov
}

Single atom catalysts (SACs) have garnered significant interest over the last decade, in part due to efforts to maximize the utilization of costly precious metal catalysts such as the Pt used in polymer electrolyte membrane fuel cells to catalyze the oxygen reduction reaction (ORR) [1]. However, due to the poor durability of Pt SACs, efforts have more recently turned towards developing suitable Pt group metal-free (PGM-free) catalysts. Amid these efforts, it was discovered that atomically dispersed transition metals have a high affinity towards the ORR. The nature of the active site has been shown to be single transition metal atoms coordinated with nitrogen within or at the edges of graphitic layers [2]. Recent improvements in performance and durability have been achieved by pyrolyzing metal organic frameworks (MOFs) doped with transition metals such as Fe, Co, and Mn [3,4].

Further improvements in mass acivity require an increase in the active site density. However, as shown in Figure 1, simply increasing the concentration of the transition metal does not result in an increase in the number of active sites; rather, excess dopants lead to the formation of metal clusters and nanoparticles, which do not contribute towards the ORR. To improve the active site density, a better understanding of the formation mechanisms of the highly active Fe-N moieties is needed. To this end, low-voltage, aberration-corrected scanning transmission electron microscopy (STEM) coupled with quantitative analytical techniques such as electron energy loss spectroscopy (EELS) and energy dispersive X-ray spectroscopy (EDS) have been employed to study the structural evolution of MOFderived catalysts at various stages of synthesis and fuel cell testing. Micro-electro-mechanical system (MEMS)-based heating devices were used to directly observe atomic-scale transformations as the temperature was increased to $1100^{\circ} \mathrm{C}$ in the STEM (Nion UltraSTEM operated at $60 \mathrm{kV}$ ).

In situ experiments revealed that significant amounts of the $\mathrm{Fe}$ and $\mathrm{N}$ sublime as the pyrolysis temperature was increased from $900-1100^{\circ} \mathrm{C}$, limiting the active site density. Further, correlative in situ X-ray adsorption spectroscopy (XAS) studies (Advanced Photon Source, Argonne National Laboratory) showed that Fe-N bonding occurred at relatively low temperatures $\left(500-600^{\circ} \mathrm{C}\right)$ and $\mathrm{Fe}$-carbides likely form at temperatures above $900^{\circ} \mathrm{C}$. Both conclusions suggest that lowering the peak pyrolysis temperature should improve active site density and mass activity. However, rotating disk electrode measurements indicate that pyrolysis temperatures above $1000^{\circ} \mathrm{C}$ are required to maximize mass activity. Additional in situ experiments revealed that the degree of carbon graphitization increased as the temperature was elevated from $900^{\circ} \mathrm{C}$ to $1100^{\circ} \mathrm{C}$, as shown by the EEL spectra in Figure 2. Graphitization of the MOF plays a critical role in the formation of the active sites and approaches are being explored to maintain higher $\mathrm{Fe}$ and $\mathrm{N}$ contents while also generating graphitized domains in the support [5]. 


\section{References:}

[1] XF Yang et al., Accounts of Chemical Research 46 (2013), p. 1740.

[2] HT Chung et al., Science 357 (2017), p. 479.

[3] XX Wang et al., Advanced Materials 48 (2018), p. 217.

[4] J Li et al., Nature Catalysis 1 (2018), p. 935.

[5] Research sponsored by the Fuel Cell Technologies Office, Office of Energy Efficiency and Renewable Energy, U.S. Department of Energy (DOE) and ORNL's Center for Nanophase Materials Sciences (CNMS), which is a U.S. DOE Office of Science User Facility
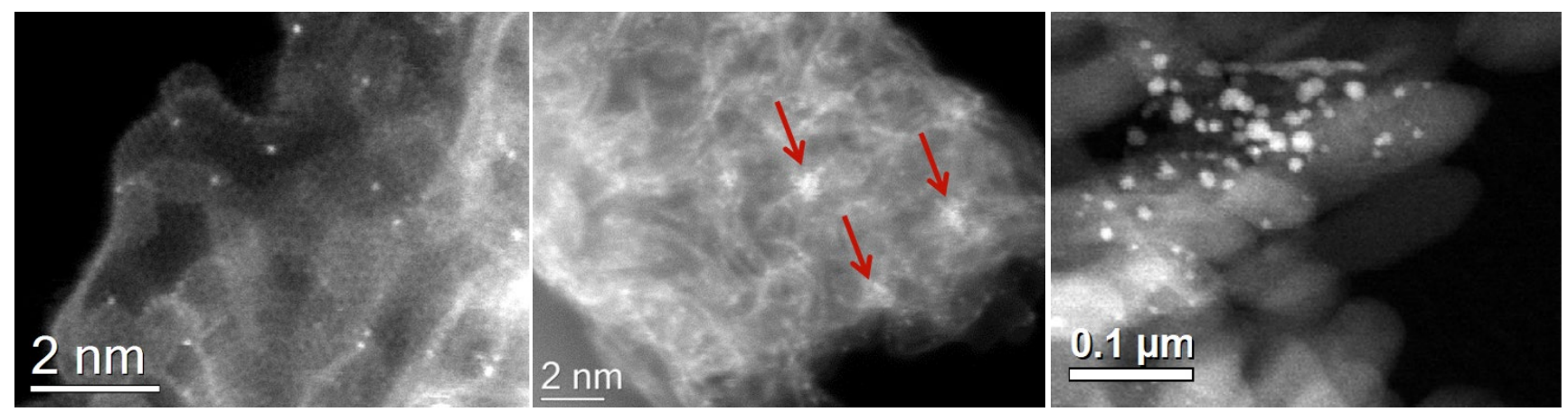

Figure 1. HAADF-STEM images of Fe-based PGM-free catalysts showing how (left) atomic dispersion gives way to (middle) metal clustering and (right) nanoparticle formation with an increase in transition metal loading.
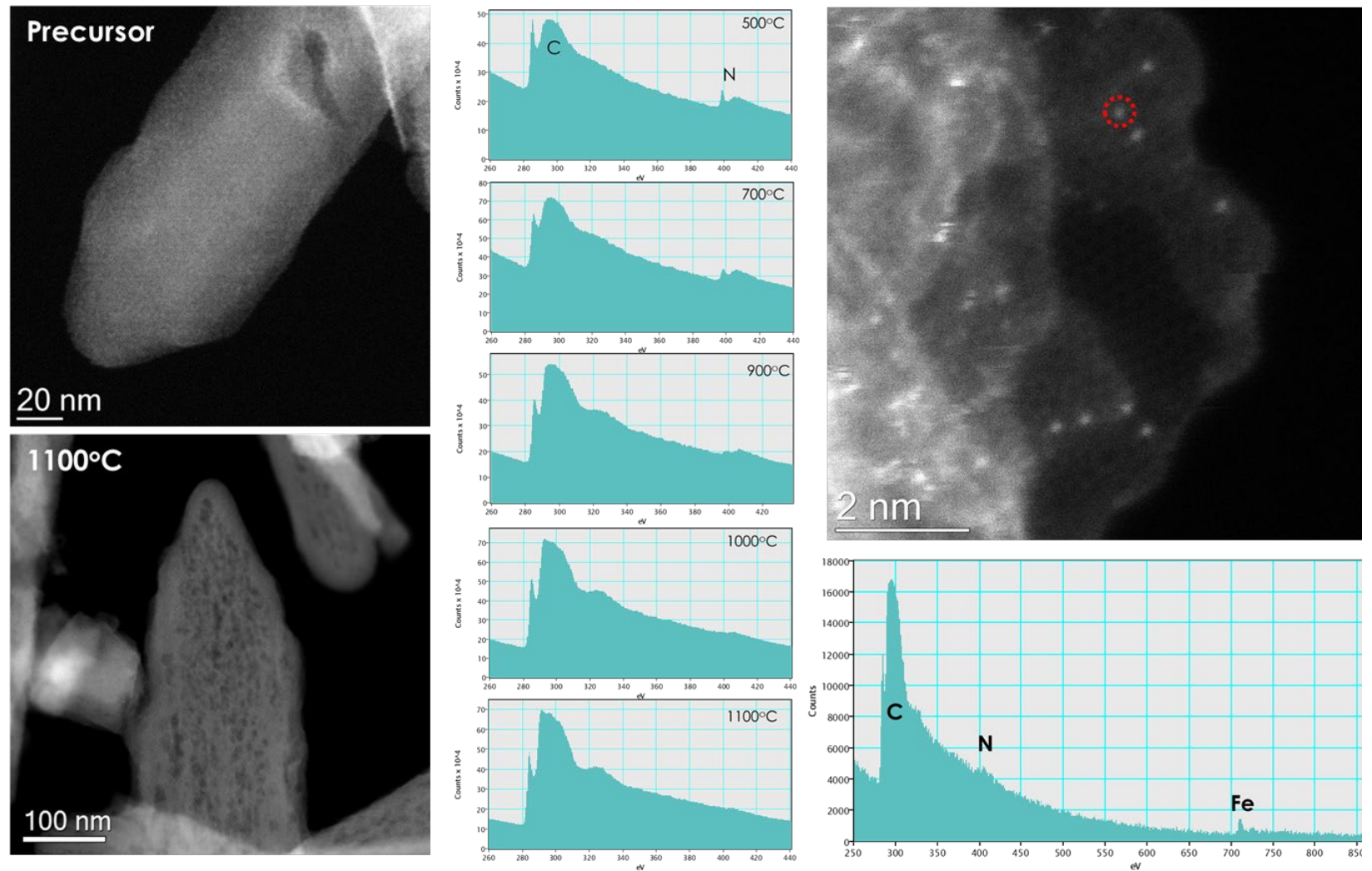

Figure 2. (left) HAADF-STEM images of precursor and pyrolyzed catalyst with high porosity. (middle) EEL spectra showing increase in degree of graphitization of carbon support with increasing pyrolysis temperature. (right) HAADF-STEM image with EEL spectrum showing co-location of $\mathrm{N}$ with Fe as marked by red circle. 